\title{
Resistin Enhances Monocyte Chemoattractant Protein-1 Production in Human Synovial Fibroblasts and Facilitates Monocyte Migration
}

\author{
Wei-Cheng Chen ${ }^{a, b}$ Shih-Wei Wang ${ }^{c, d}$ Chih-Yang Lin ${ }^{c} \quad$ Chun-Hao Tsaie,f \\ Yi-Chin Fong ${ }^{e, g}$ Ting-Yi Lin ${ }^{b}$ Shun-Long Weng ${ }^{c, h}$ Hsien-Da Huang ${ }^{i, j, k}$ \\ Kuang-Wen Liao ${ }^{a, 1, m, n}$ Chih-Hsin Tang ${ }^{o, p, q, r}$
}

aPh.D. Degree Program of Biomedical Science and Engineering, National Chiao Tung University, Hsinchu, Taiwan, bDepartment of Orthopaedics, MacKay Memorial Hospital, Taipei, Taiwan, 'Department of Medicine, Mackay Medical College, New Taipei City, Taiwan, 'dGraduate Institute of Natural Products, College of Pharmacy, Kaohsiung Medical University, Kaohsiung, Taiwan, eDepartment of Sports Medicine, College of Health Care, China Medical University, Taichung, Taiwan, 'Department of Orthopedic Surgery, China Medical University Hospital, Taichung, Taiwan, 9Department of Orthopedic Surgery, China Medical University Beigang Hospital, Yunlin, Taiwan, hDepartment of Obstetrics and Gynaecology, Hsinchu MacKay Memorial Hospital, Hsinchu, Taiwan, 'Warshel Institute For Computational Biology, The Chinese University of Hong Kong, Shenzhen, China, ischool of Life

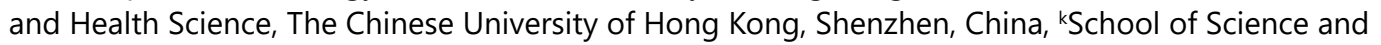
Engineering, The Chinese University of Hong Kong, Shenzhen, China, 'Department of Biological Science and Technology, National Chiao Tung University, Hsinchu, Taiwan, mInstitute of Molecular Medicine and Bioengineering, National Chiao Tung University, Hsinchu, Taiwan, "Department of Biotechnology and Bioindustry Sciences, National Cheng Kung University, Tainan, Taiwan, ${ }^{\circ}$ Department of Pharmacology, School of Medicine, China Medical University, Taichung, Taiwan, ${ }^{\mathrm{P} C h i n e s e ~ M e d i c i n e ~ R e s e a r c h ~ C e n t e r, ~}$ China Medical University, Taichung, Taiwan, "Graduate Institute of Basic Medical Science, China Medical University, Taichung, Taiwan, 'Department of Biotechnology, College of Health Science, Asia University, Taichung, Taiwan

\section{Key Words}

Osteoarthritis • Resistin $\cdot \mathrm{MCP}-1 \cdot$ Monocyte migration

\begin{abstract}
Background/Aims: The adipocyte-secreting adipokine, resistin, may play a critical role in the modulation of inflammatory diseases. Migration and infiltration of mononuclear cells into inflammatory sites are critical events during the development of osteoarthritis (OA). Monocyte chemoattractant protein-1 (MCP-1), also known as chemokine ligand 2 (CCL2),

Chih-Hsin Tang, PhD

and Kuang-Wen Liao, PhD

Department of Pharmacology, School of Medicine, China Medical University

No. 91, Hsueh-Shih Road, Taichung (Taiwan)

Tel. (886) 4-22052121 Ext. 7726, E-Mail chtang@mail.cmu.edu.tw; liaonms@pchome.com.tw
\end{abstract}




\section{Cellular Physiology Cell Physiol Biochem 2019;52:408-420 \\ \begin{tabular}{ll|l} 
and Biochemistry $\begin{array}{l}\text { DOl: 10.33594/000000029 } \\
\text { Published online: } 8 \text { March 2019 }\end{array}$ & $\begin{array}{l}\text { O } 2019 \text { The Author(s). Published by } \\
\text { Cell Physiol Biochem Press GmbH\&Co. KG }\end{array}$ \\
\cline { 2 - 3 }
\end{tabular} \\ Chen et al.: Resistin Enhances MCP-1-Mediated Monocyte Migration}

plays a critical role in the regulation of monocyte migration and infiltration. In this study, we show how resistin promotes MCP-1 expression in OA synovial fibroblasts and monocyte migration. Methods: We used qPCR to detect MCP-1 and miRNA expression. THP-1 migration was investigated by Transwell assay. The Western blotting was used to examine the resistinmediated signaling pathways. Results: Resistin activated the phosphatidylinositol-3-kinase (PI3K), Akt and mammalian target of rapamycin (mTOR) signaling pathways, while PI3K, Akt and mTOR inhibitors or small interfering RNAs diminished resistin-induced MCP-1 expression and monocyte migration. We also demonstrate that resistin stimulates MCP-1mediated monocyte migration by suppressing microRNA (miR)-33a and miR-33b via the $\mathrm{PI3K}$, Akt and mTOR signaling pathways. Conclusion: These results provide new insights into the mechanisms of resistin action that may have therapeutic implications for patients with OA.

(c) 2019 The Author(s). Published by

Cell Physiol Biochem Press GmbH\&Co. KG

\section{Introduction}

Osteoarthritis $(\mathrm{OA})$ is the most common form of arthritis and the single most important determinant of disability in older adults [1]. No specific cause has been identified for OA, although aging, gender, obesity, genetic factors and injury all increase the risk of OA $[2$, 3]. Inflammation, even in the early stage of the disease, is believed to be involved in $\mathrm{OA}$ progression and development [4]. Synovial membrane inflammation is also implicated in the disease pathophysiology. Biochemical mediators such as interleukins, cytokines, chemokines and growth factors in OA synovial fibroblasts (OASFs) affect knee joint cellular functioning [5]. In particular, cytokines and chemokines enhance inflammation, neovascularization and cartilage degradation, which facilitates OA progression [6].

Increasing evidence indicates migration and infiltration of mononuclear cells to inflammatory sites are regulated by adhesion molecules, such as monocyte chemoattractant protein-1 (MCP-1), also known as the chemokine (C-C motif) ligand 2 (CCL2) [7, 8]. MCP1 initiates chemotaxis and transendothelial migration of monocytes to inflammatory lesions [9]. In various inflammatory conditions such as arthritis [10], multiple sclerosis, atherosclerosis [11] and cancer [12], MCP-1 attracts monocytes and lymphocytes to the site of inflammation. OA and rheumatoid arthritis (RA) are characterized by high levels of MCP-1 expression in the blood, synovial fluid and synovial tissue [13-15]. In preclinical investigations, increased production of MCP-1 promoted the development of synovitis [16], and macrophage infiltration was markedly increased after MCP-1 injections into rabbit joints [14]. MCP-1 is thought to act as a proinflammatory agent and increase monocyte expression in synovial fluid and tissue during the OA disease process [17-19].

Resistin is a 12.5 -kDa cysteine-rich adipokine that is constitutively secreted by adipose tissue [20]; resistin levels in plasma correlate with inflammatory markers and coronary artery calcification, a measure of coronary atherosclerosis [21]. Moreover, resistin promotes VEGF expression and angiogenesis in EPCs in the pathogenesis of RA [22] and resistin single nucleotide polymorphisms have been linked to RA susceptibility and clinicopathological characteristics [23]. Elevated resistin may be a predictor of OA: patients with OA have high levels of resistin levels in serum and synovial fluid [24] and resistin levels might correlate with markers of inflammation.

Resistin promotes an inflammatory response in OA and RA [7, 25]. However, although it is recognized that resistin is involved in OA pathogenesis, the role of resistin in MCP-1 expression and monocyte accumulation is unclear. Our study shows that resistin upregulates MCP-1 expression in OASFs and promotes monocyte migration. We also show that resistininduced downregulation of miR-33a/miR-33b involves the PI3K, Akt and mammalian target of rapamycin (mTOR) signaling pathways. These results provide new therapeutic insights into the mechanisms of resistin in OA. 


\section{Cellular Physiology Cell Physiol Biochem 2019;52:408-420 \begin{tabular}{ll|l} 
and Biochemistry & $\begin{array}{l}\text { DOl: 10.33594/000000029 } \\
\text { Published online: } 8 \text { March 2019 }\end{array}$ & $\begin{array}{l}\text { @ } 2019 \text { The Author(s). Published by } \\
\text { Cell Physiol Biochem Press GmbH\&Co. KG }\end{array}$ \\
\cline { 2 - 3 }
\end{tabular} \\ Chen et al.: Resistin Enhances MCP-1-Mediated Monocyte Migration}

\section{Materials and Methods}

\section{Materials}

Recombinant human resistin was purchased from R\&D Systems (Minneapolis, MN, USA). We purchased p85, Akt and mTOR primary antibodies (Santa Cruz Biotechnology, CA, USA), as well as rabbit polyclonal antibodies specific for p-p85, p-Akt and p-mTOR (Cell Signaling Technology, Danvers, MA, USA). miR-33a and miR-33b mimics, miRNA control, Lipofectamine 2000, and Trizol were purchased from Life Technologies (Carlsbad, CA, USA). Dharmacon Research (Lafayette, CO, USA) supplied ON-TARGETplus ${ }^{\text {tw }}$ siRNAs. GibcoBRL life technologies (Grand Island, NY, USA) supplied fetal bovine serum (FBS), RPMI-1640, $\alpha$-MEM, and all other cell culture reagents. All other chemicals or inhibitors were purchased from Sigma-Aldrich (St. Louis, MO, USA).

\section{Cell culture}

Human synovial fibroblasts were isolated by collagenase treatment of synovial tissue samples obtained from 6 patients with OA undergoing knee replacement surgery in China Medical University Hospital. All study participants gave written consent before enrollment. The study protocol was approved by China Medical University Hospital's Institutional Review Board. OASFs were isolated, cultured, and characterized as previously described [26, 27]. Experiments were performed using cells from passages 3 to 6 .

THP-1, a human leukemia cell line of monocyte/macrophage lineage, was obtained from The American Type Culture Collection (Manassas, VA, USA) and grown in RPMI-1640 medium with 10\% FBS.

Quantitative real-time PCR ( $q P C R$ ) of $m R N A$ and miRNA

Total RNA was extracted from OASFs cells using TRIzol reagent. qPCR analysis was carried out according to an established protocol $[28,29]$.

\section{Western blot analysis}

Cell lysates underwent electrophoresis with SDS-PAGE and were transferred to PVDF membranes according to the method described in our previous studies [30,31]. After blocking the blots with $4 \%$ bovine serum albumin, we treated them with primary antibody and then peroxidase-conjugated secondary antibody. The blots were visualized using the chemiluminescence UVP BioSpectrum system (UVP, Upland, CA, USA).

In vitro chemotaxis assay

Conditioned medium obtained from resistin-treated OASFs were placed in the lower compartment of the Transwell assay (Costa Corning, Cambridge, MA, USA). THP-1 cells (5 x $10^{4}$ cells) were added to the upper chamber and cell migratory ability was assayed using the method described in our previous work $[8,32]$.

\section{Statistical Analysis}

Data are presented as mean \pm standard error of the mean (SEM). The Student's $t$-test determined statistical differences between samples and the Bonferroni post hoc procedure was performed for a oneway analysis of variance (ANOVA) of statistical comparisons between more than two samples; $p$-values of less than 0.05 were considered to be statistically significant.

\section{Results}

Resistin promotes MCP-1 production in human synovial fibroblasts and facilitates monocyte migration

MCP-1 is indicated to be a critical chemokine that regulates monocyte migration and infiltration [9]. In this study, directly applying resistin to OASFs promoted mRNA expression of MCP-1 in a concentration-dependent manner (Fig. 1A). We next investigated whether resistin-induced MCP-1 expression facilitates monocyte migration. An in vitro chemotaxis assay revealed that conditioned medium from resistin-treated OASFs increased monocyte 
migration, while incubation with MCP-1 neutralizing antibody abolished resistin-promoted monocyte migration (Fig. 1B). These results suggest that resistin induces MCP-1 expression in synovial fibroblasts and thereby promotes monocyte migration.

The PI3K/Akt signaling pathway plays a role in resistin-induced MCP-1 expression and monocyte migration

The PI3K/Akt signaling pathway is frequently implicated in inflammatory responses [33]. We found that pretreating OASFs with PI3K inhibitors (Ly294002, wortmannin) or an Akt inhibitor abolished resistin-enhanced MCP-1 expression (Fig. 2A\&3A). These inhibitors also diminished resistin-induced migration of monocytes (Fig. 2C\&3C). PI3K and Akt siRNA showed similar effects (Fig. 2B\&D and 3B\&D). The PI3K-dependent signaling pathway activates Akt residue phosphorylation [34]. We identified a significant, time-dependent induction of p85 and Akt phosphorylation in response to resistin treatment (Fig. 2E\&3E). Pretreating the cells with a PI3K inhibitor blocked resistin-induced Akt phosphorylation (Fig. 3F). It appears that resistin acts via the PI3K/Akt-dependent signaling pathway to increase MCP-1 expression and promote monocyte migration.

Resistin increases MCP-1 production and facilitates monocyte migration via the mTOR pathway

mTOR activation is a common downstream event of the PI3/Akt signaling pathway [35]. In our investigation, resistin-induced MCP- 1 mRNA expression and monocyte migration was reduced by an mTOR inhibitor and an siRNA (Fig. 4A-D). In addition, resistin increased mTOR phosphorylation in OASFs (Fig. 4E), which was antagonized when cells were incubated with PI3K or Akt inhibitors (Fig. 4F). Thus, the PI3K/Akt/mTOR signaling pathway mediates the effects of resistin upon MCP-1 expression and monocyte migration.

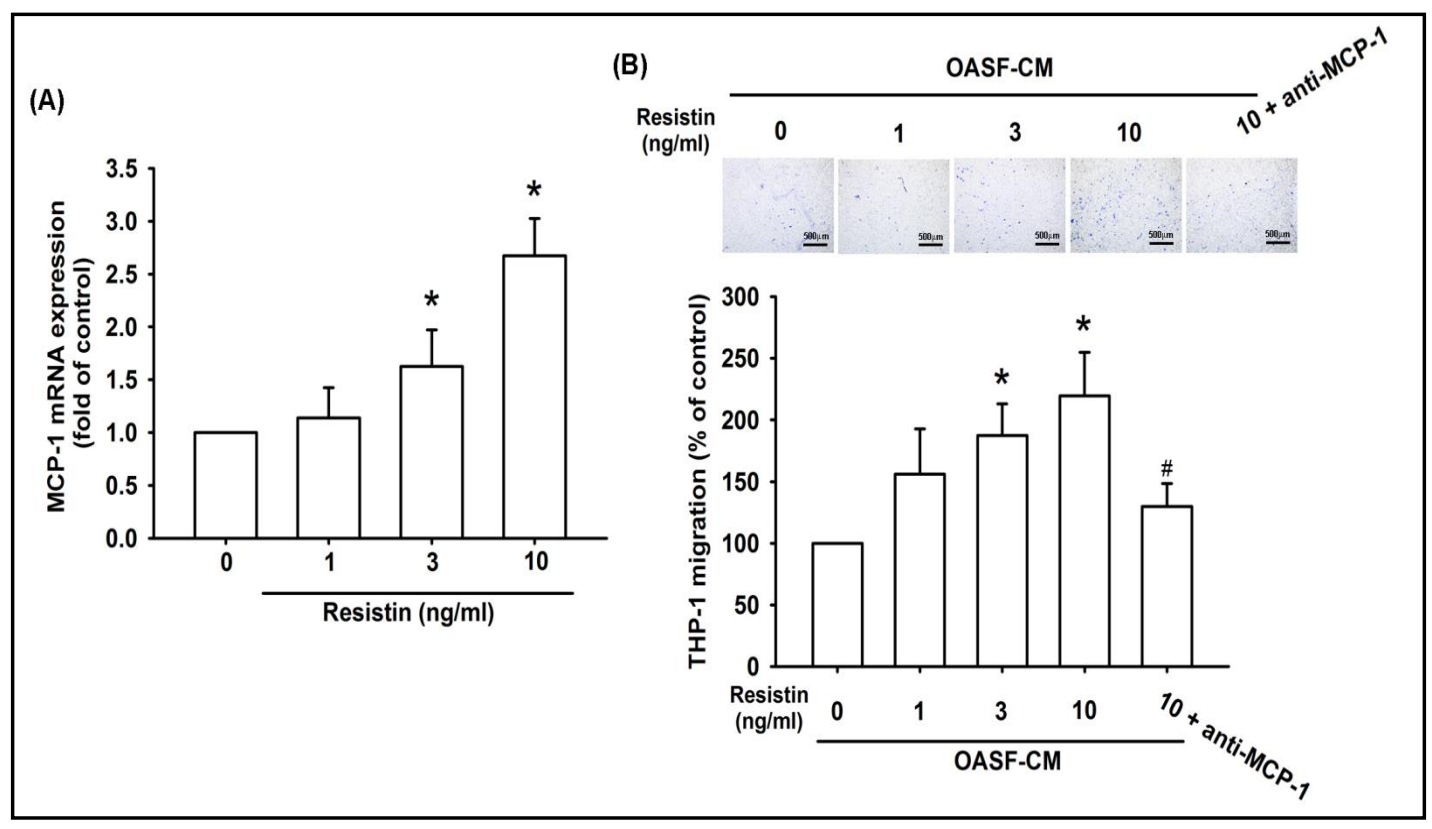

Fig. 1. Resistin promotes MCP-1 expression in OASFs and facilitates monocyte migration. (A) OASFs were incubated with resistin (1-10 ng/ml) for $24 \mathrm{~h}$; MCP-1 mRNA expression was examined by qPCR. (B) OASFs were incubated with resistin for $24 \mathrm{~h}$ then stimulated with MCP-1 antibody $(1 \mu \mathrm{g} / \mathrm{ml})$ for $30 \mathrm{~min}$. The conditioned medium (CM) was then collected and applied to THP-1 cells. THP-1 migration was measured. Results are expressed as the mean \pm SEM. ${ }^{*} \mathrm{p}<0.05$ as compared with the control group; $\# \mathrm{p}<0.05$ as compared with the resistin-treated group. 
(A)

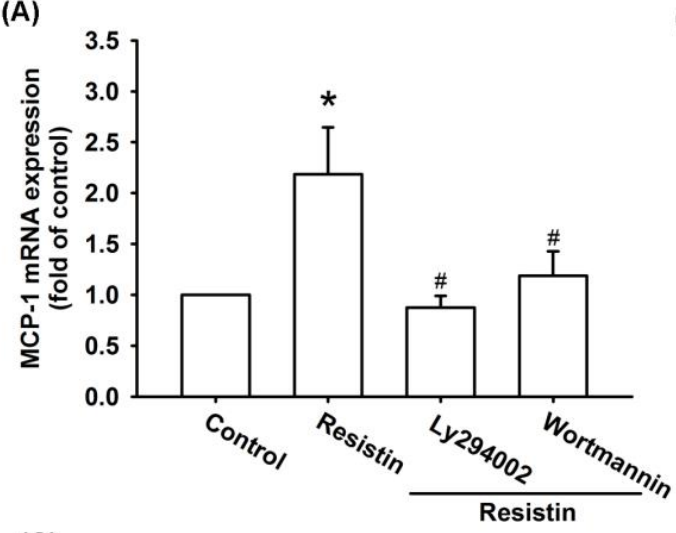

(C)

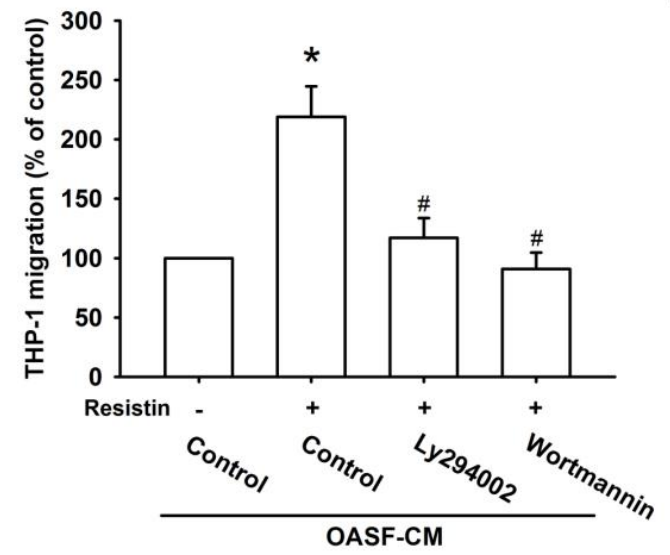

(E)

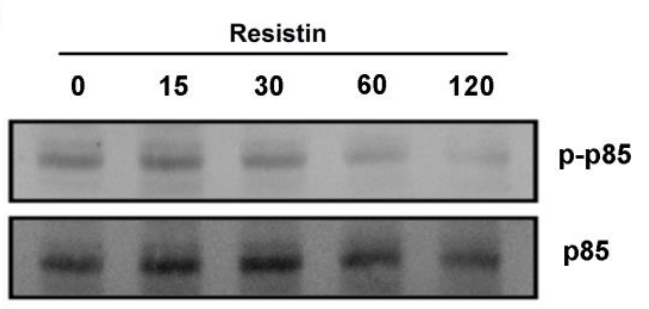

(B)

(D)
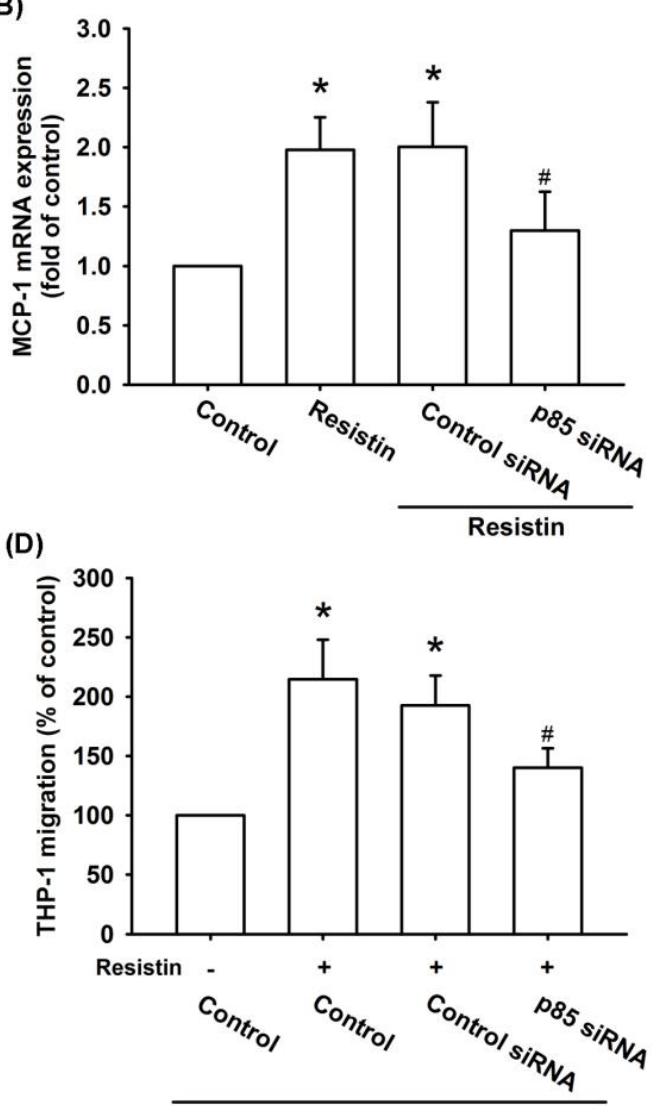

OASF-CM

Fig. 2. The PI3K pathway is involved in resistin-induced MCP-1 expression and monocyte migration. (A\&B) OASFs were pretreated for 30 min with Ly294002 $(10 \mu \mathrm{M})$ and wortmannin $(5 \mu \mathrm{M})$ or transfected with p85 siRNA, then stimulated with resistin. MCP-1 expression was examined by qPCR. (C\&D) CM was applied to THP-1 cells and analyzed for migration activity. (E) OASFs were incubated with resistin and p-p85 expression was examined by Western blot. Results are expressed as the mean \pm SEM. ${ }^{*} \mathrm{p}<0.05$ as compared with the control group; $\# \mathrm{p}<0.05$ as compared with the resistin-treated group.

Resistin facilitates MCP-1-related monocyte migration by suppressing miR-33a/miR-33b

Accumulating evidencesuggests thatmiRNAsare crucial regulators of cellmotility $[33,36]$. Using open-source software, we found that the 3'UTR region of MCP-1 mRNA harbors potential binding sites for 5 candidate miRNAs, and that miR-33a (GTGCATTGTAGTTGCATTGCA) and miR-33b (GTGCATTGCTGTTGCATTGC) are markedly downregulated after resistin treatment (Fig. 5A). Exogenous resistin concentration-dependently inhibited miR-33a and miR-33b expression (Fig. 5B). Transfection of cells with miR-33a and miR-33b mimics diminished resistin-enhanced MCP-1 expression (Fig. 5C) and also inhibited resistin-boosted monocyte migration (Fig. 5D). 


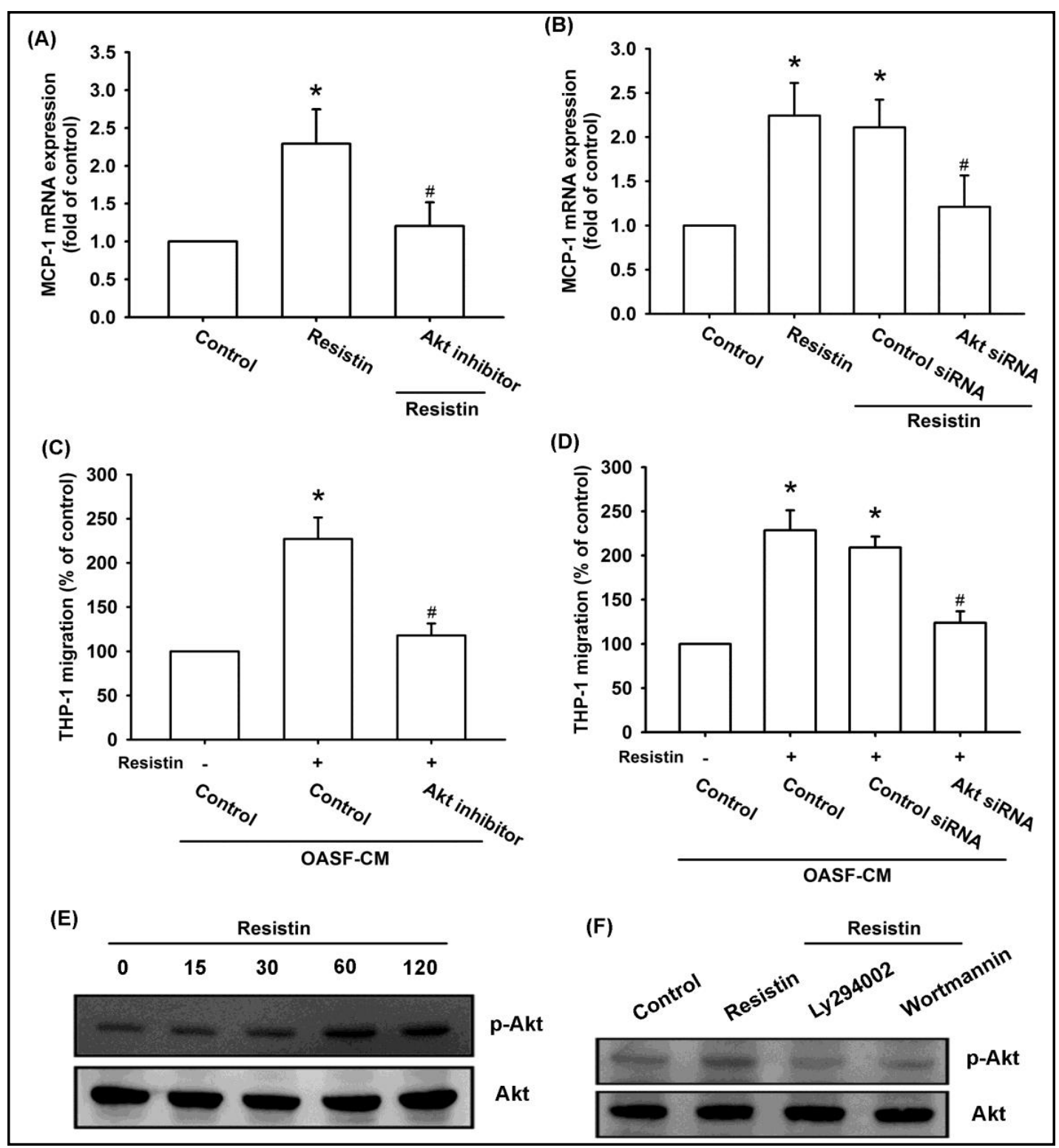

Fig. 3. Akt is involved in resistin-induced MCP-1 expression and monocyte migration. (A\&B) OASFs were pretreated for 30 min with an Akt inhibitor $(10 \mu \mathrm{M})$ or transfected with the Akt siRNA then stimulated with resistin. MCP-1 expression was examined by qPCR. (C\&D) CM was applied to THP-1 cells and analyzed for migration activity. (E\&F) OASFs were incubated with resistin or pretreated with PI3K inhibitors then stimulated with resistin and p-Akt expression was examined by Western blot. Results are expressed as the mean \pm SEM. ${ }^{*} \mathrm{p}<0.05$ as compared with the control group; $\# \mathrm{p}<0.05$ as compared with the resistin-treated group.

Next, we examined the relationship between the PI3K/Akt/mTOR pathway and miR33a/miR-33b. Resistin-induced reductions in miR-33a and miR-33b expression were reversed when the cells were treated with PI3K, Akt and mTOR inhibitors (Fig. 6A\&C\&E) and also by PI3K, Akt and mTOR siRNAs (Fig. 6B\&D\&F), indicating that resistin induces MCP1 expression and monocyte migration by inhibiting miR-33a/miR-33b via the PI3K, Akt and mTOR pathways. 
(A)

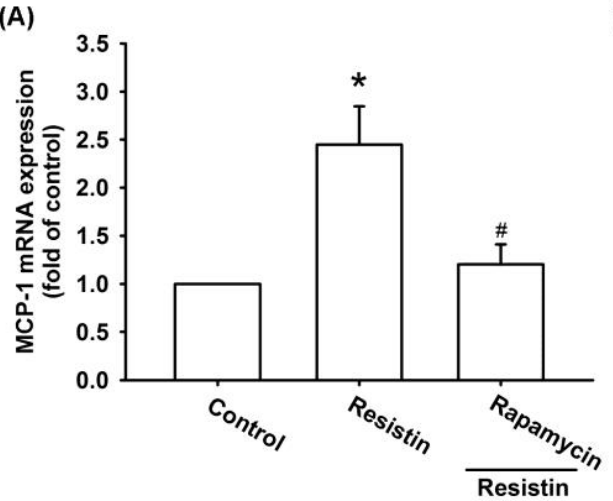

(C)

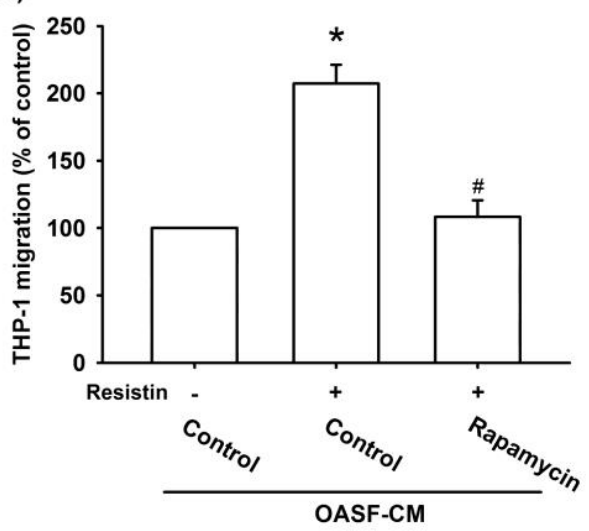

(E)

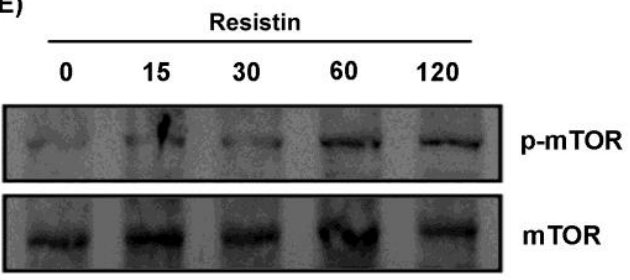

(B)

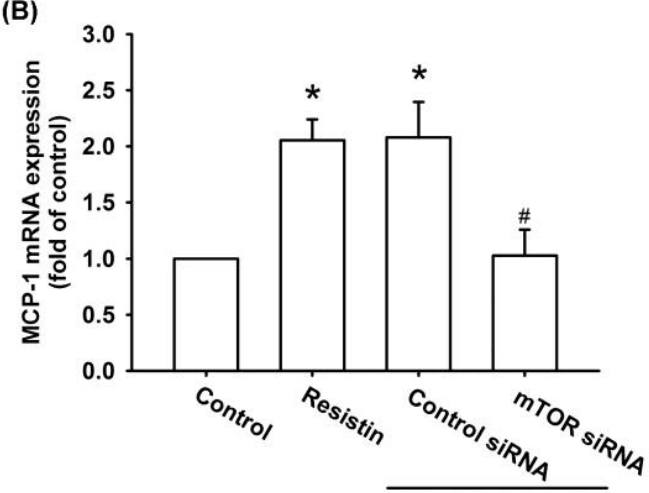

(D)

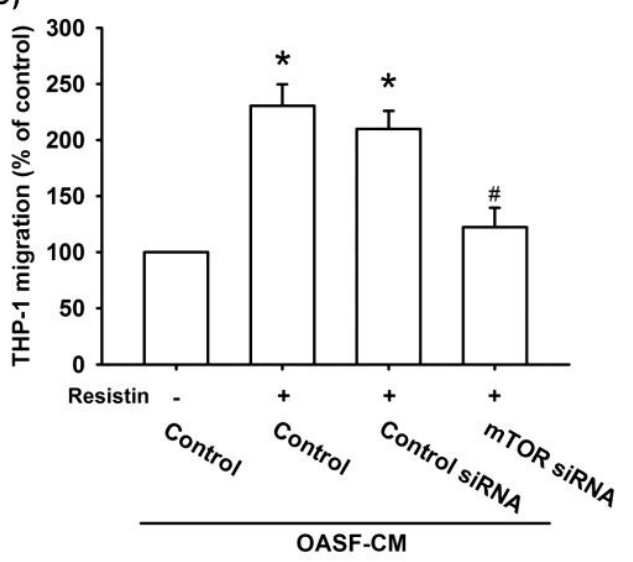

(F)

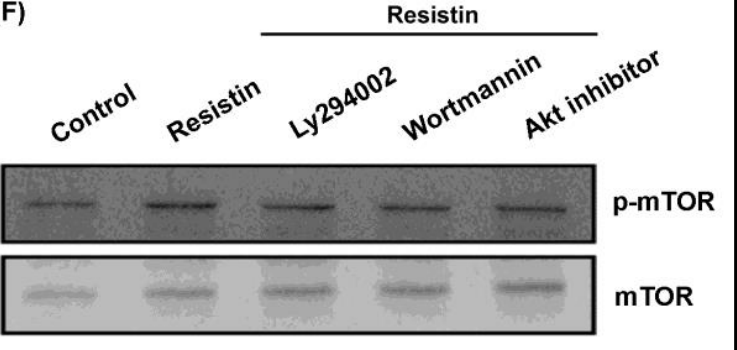

Fig. 4. $\mathrm{mTOR}$ is involved in resistin-induced MCP-1 expression and monocyte migration. (A\&B) OASFs were pretreated for $30 \mathrm{~min}$ with rapamycin $(10 \mu \mathrm{M})$ or transfected with the mTOR siRNA then stimulated with resistin. MCP-1 expression was examined by qPCR. (C\&D) CM was applied to THP-1 cells and analyzed for migration activity. (E\&F) OASFs were incubated with resistin or pretreated with PI3K and Akt inhibitors then stimulated with resistin and p-mTOR expression was examined by Western blot. Results are expressed as the mean \pm SEM. ${ }^{*} \mathrm{p}<0.05$ as compared with the control group; $\# \mathrm{p}<0.05$ as compared with the resistintreated group.

\section{Discussion}

OA pathogenesis is little understood. It is well accepted, however, that effective immune surveillance involves the monitoring of leukocyte infiltration to sites of inflammatory stimuli. MCP-1 is the main regulator of monocyte infiltration into inflammatory sites during $\mathrm{OA}$ pathogenesis. Resistin is an adipokine that is associated with obesity, inflammation, and various cancers [22, 25, 37]. High levels of resistin have been found in serum and joint fluids of $\mathrm{OA}$ and RA patients $[24,25]$. We hypothesized that resistin would influence monocyte 


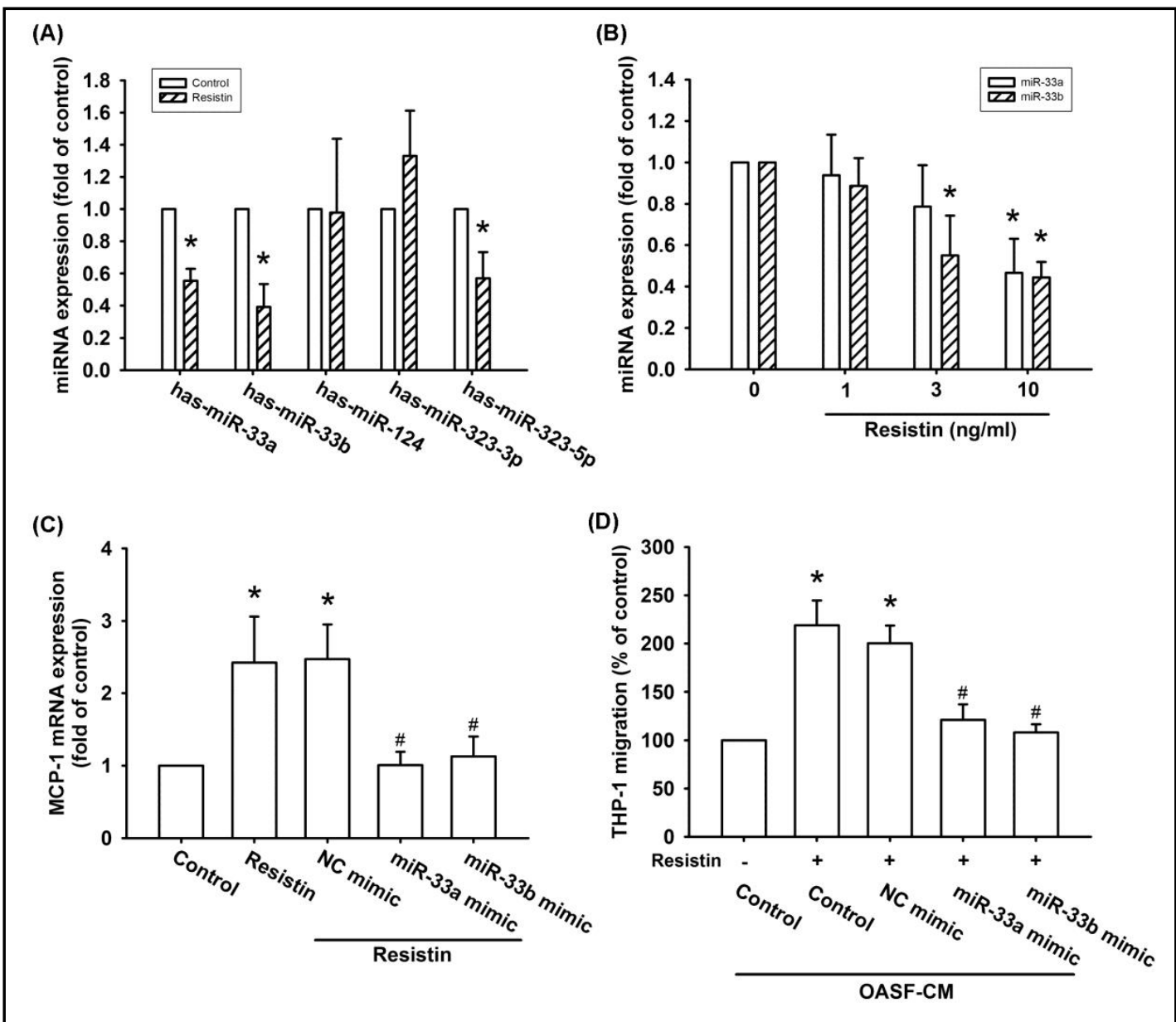

Fig. 5. Resistin promotes MCP-1-mediated monocyte migration by inhibiting miR-33a and miR-33b. (A\&B) OASFs were incubated with resistin for $24 \mathrm{~h}$ and miRNA expression was examined by qPCR. (C) OASFs were transfected with the miR-33a and miR-33b mimics then incubated with resistin for $24 \mathrm{~h}$; MCP-1 expression was measured by qPCR. (D) CM was applied to THP-1 cells and analyzed for migration activity. Results are expressed as the mean \pm SEM. ${ }^{*} \mathrm{p}<0.05$ as compared with the control group; $\# \mathrm{p}<0.05$ as compared with the resistin-treated group.

infiltration during OA pathogenesis. In this study, we provide evidence showing that resistin induces MCP-1 production in human OASFs and contributes to monocyte migration by suppressing miR-33a/miR-33b expression through the PI3K/Akt/mTOR signaling pathway (Fig. 7). This is the first indication that adipokine resistin boosts MCP-1-associated monocytes migration via the downregulation of miR-33a/miR-33b. Similar effects have been reported with other adipokines, such as adiponectin, which increases IL- 6 and ICAM-1 expression during the development of arthritis [38, 39], while leptin enhances IL-8 and oncostatin M production in arthritis [40,41]. In addition, the adipocyte hormone visfatin upregulates levels of IL- 6 and TNF- $\alpha$ expression in arthritis disease [27]. These findings suggest that adipokines play a pro-inflammatory role in arthritis.

Activation of the PI3K/Akt signaling pathway is a critical event in inflammatory responses and represents a potential therapeutic target against inflammatory disorders. This pathway mediates multiple cellular functions, including cell survival, proliferation, migration, and autophagy [42]. In this study, we demonstrated that PI3K and Akt inhibitors are capable of inhibiting resistin-induced MCP-1 expression and monocyte migration. Furthermore, we observed that p85 and Akt siRNAs reduced MCP-1 expression in OASFs as well as monocyte migration. Incubation of cells with resistin increased PI3K and Akt phosphorylation. The 


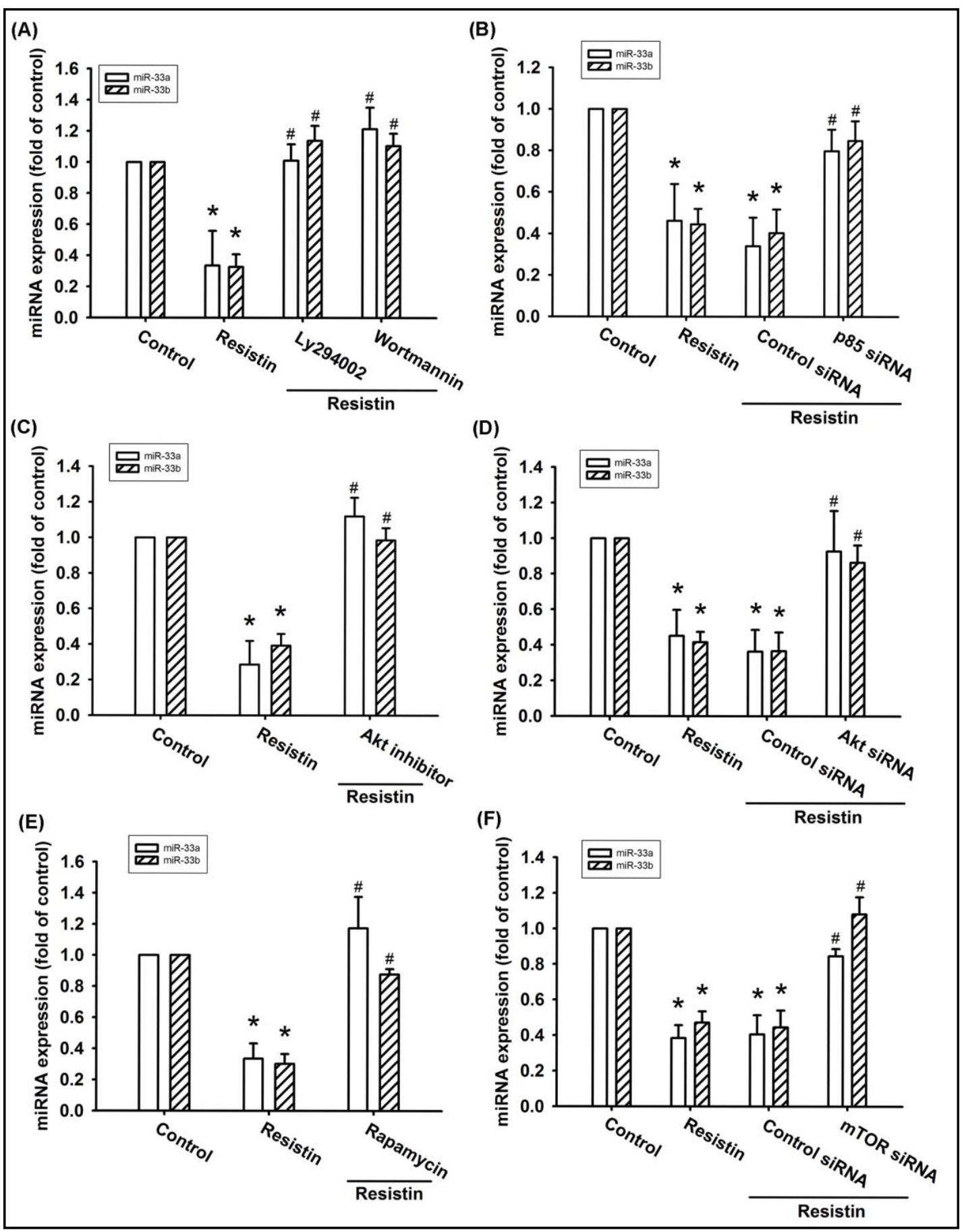

Fig. 6. Resistin inhibits miR-33a and miR-33b expression through the PI3K, Akt and mTOR pathways. OASFs were pretreated for 30 min with PI3K, Akt and mTOR inhibitors or siRNAs, then stimulated with resistin. miRNA expression was examined by qPCR. Results are expressed as the mean \pm SEM. ${ }^{*} \mathrm{p}<0.05$ as compared with the control group; $\# \mathrm{p}<0.05$ as compared with the resistin-treated group.

resistin-induced increase in Akt phosphorylation was suppressed when cells were pretreated with a PI3K inhibitor. This indicates that the PI3K/Akt signaling pathway is involved in resistin-mediated MCP-1 expression in OASFs and monocyte migration.

Increasing reports have shown that mTOR is a major downstream signaling molecule of the PI3K/Akt pathway $[43,44]$. We therefore examined the role of mTOR in resistin- 
mediated MCP-1 production and monocyte migration. We found that an mTOR inhibitor (rapamycin) reduced resistinpromoted MCP-1 expression in OASFs. Furthermore, resistinenhanced MCP-1 production and monocyte migration were diminished by treatment with an mTOR inhibitor and an siRNA. We also observed that mTOR phosphorylation was increased after treating OASFs with resistin. Stimulation of cells with PI3K or Akt inhibitors reduced resistinpromoted phosphorylation of mTOR, suggesting that resistin promotes MCP-1 production in OASFs and subsequently promotes monocyte migration through the PI3K/Akt/mTOR signaling cascades.

miRNAs inhibit and

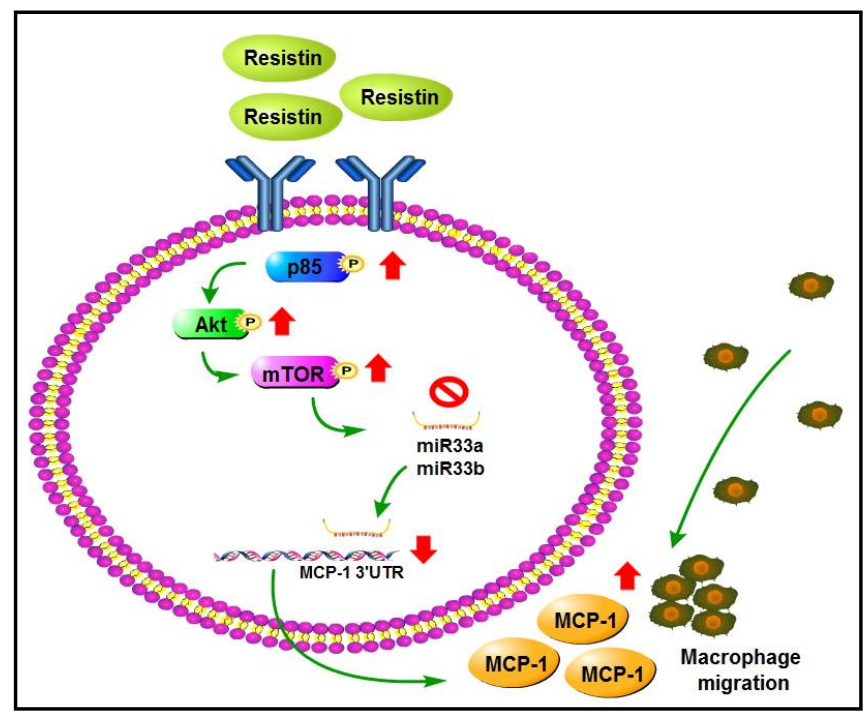

Fig. 7. Schematic diagram summarizes the mechanism of resistin-promoted MCP-1 expression and boosted monocyte migration. Resistin induces MCP-1 production in OASFs by downregulating miR-33a/miR33b through the PI3K/Akt/mTOR pathway and subsequently facilitates monocyte migration. regulate gene expression [45]. In particular, they help to regulate various inflammatory diseases, including OA and RA [46]. We therefore sought to examine whether miRNAs are implicated in MCP-1 expression following resistin treatment. Our results demonstrate that resistin significantly reduces miR-33a and miR-33b expression in OASFs, while transfecting OASFs with miR-33a and miR-33b mimics antagonizes resistin-enhanced MCP-1 expression and monocyte migration. Strikingly, treatment with PI3K, Akt and mTOR inhibitors or an siRNA reversed resistin-inhibited miR$33 \mathrm{a}$ and miR-33b expression, indicating that resistin promotes MCP-1-mediated monocyte migration by suppressing miR-33a and miR-33b expression through the PI3K, Akt and mTOR signaling cascades.

\section{Conclusion}

This study has identified that resistin increases MCP-1 production in OASFs by suppressing miR-33a/miR-33b expression through the PI3K/Akt/mTOR signaling cascades, facilitating monocyte migration. Resistin may be a novel therapeutic target in OA.

\section{Acknowledgements}

This work was supported by grants from Taiwan's Ministry of Science and Technology (MOST 105-2320-B-039-015-MY3), China Medical University (CMU106-S-27), MacKay Memorial Hospital (MMH-107-55, MMH-108-53), Mackay Medical College (MMC-1071B27).

\section{Disclosure Statement}

The authors have no financial or personal relationships that could inappropriately influence this research. 


\section{Cellular Physiology Cell Physiol Biochem 2019;52:408-420

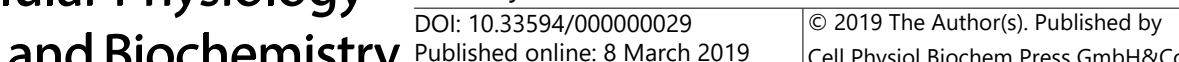 and Biochemistry Published online: 8 March 2019 Cell Physiol Biochem Press GmbH\&Co. KG \\ Chen et al.: Resistin Enhances MCP-1-Mediated Monocyte Migration}

\section{References}

- 1 Chou PY, Su CM, Huang CY, Tang CH: The characteristics of thrombin in osteoarthritic pathogenesis and treatment. Biomed Res Int 2014;2014:407518.

2 Wu Y, Goh EL, Wang D, Ma S: Novel treatments for osteoarthritis: an update. Open Access Rheumatol 2018;10:135-140.

3 Marshall M, Watt FE, Vincent TL, Dziedzic K: Hand osteoarthritis: clinical phenotypes, molecular mechanisms and disease management. Nat Rev Rheumatol 2018;14:641-656.

4 Wang T, He C: Pro-inflammatory cytokines: The link between obesity and osteoarthritis. Cytokine Growth Factor Rev 2018; DOI:10.1016/j.cytogfr.2018.10.002.

5 MacDonald IJ, Liu SC, Su CM, Wang YH, Tsai CH, Tang CH: Implications of Angiogenesis Involvement in Arthritis. Int J Mol Sci 2018;19:pii:E2012.

6 Scanzello CR, Goldring SR: The role of synovitis in osteoarthritis pathogenesis. Bone 2012;51:249-257.

7 Raghu H, Lepus CM, Wang Q, Wong HH, Lingampalli N, Oliviero F, Punzi L, Giori NJ, Goodman SB, Chu CR, Sokolove JB, Robinson WH: CCL2/CCR2, but not CCL5/CCR5, mediates monocyte recruitment, inflammation and cartilage destruction in osteoarthritis. Ann Rheum Dis 2017;76:914-922.

8 Chen CY, Fuh LJ, Huang CC, Hsu CJ, Su CM, Liu SC, Lin YM, Tang CH: Enhancement of CCL2 expression and monocyte migration by CCN1 in osteoblasts through inhibiting miR-518a-5p: implication of rheumatoid arthritis therapy. Sci Rep 2017;7:421.

9 Lin YM, Hsu CJ, Liao YY, Chou MC, Tang CH: The CCL2/CCR2 axis enhances vascular cell adhesion molecule-1 expression in human synovial fibroblasts. PLoS One 2012;7:e49999.

10 Tsou HK, Chen HT, Chang CH, Yang WY, Tang CH: Apoptosis signal-regulating kinase 1 is mediated in TNFalpha-induced CCL2 expression in human synovial fibroblasts. J Cell Biochem 2012;113:3509-3519.

- 11 Taylor PC, Peters AM, Paleolog E, Chapman PT, Elliott MJ, McCloskey R, Feldmann M, Maini RN: Reduction of chemokine levels and leukocyte traffic to joints by tumor necrosis factor alpha blockade in patients with rheumatoid arthritis. Arthritis Rheum 2000;43:38-47.

12 Tang CH, Tsai CC: CCL2 increases MMP-9 expression and cell motility in human chondrosarcoma cells via the Ras/Raf/MEK/ERK/NF-kappaB signaling pathway. Biochem Pharmacol 2012;83:335-344.

13 Koch AE, Kunkel SL, Harlow LA, Johnson B, Evanoff HL, Haines GK, Burdick MD, Pope RM, Strieter RM: Enhanced production of monocyte chemoattractant protein-1 in rheumatoid arthritis. J Clin Invest 1992;90:772-779.

14 Akahoshi T, Wada C, Endo H, Hirota K, Hosaka S, Takagishi K, Kondo H, Kashiwazaki S, Matsushima K: Expression of monocyte chemotactic and activating factor in rheumatoid arthritis. Regulation of its production in synovial cells by interleukin-1 and tumor necrosis factor. Arthritis Rheum 1993;36:762-771.

15 Stankovic A, Slavic V, Stamenkovic B, Kamenov B, Bojanovic M, Mitrovic DR: Serum and synovial fluid concentrations of CCL2 (MCP-1) chemokine in patients suffering rheumatoid arthritis and osteoarthritis reflect disease activity. Bratisl Lek Listy 2009;110:641-646.

- 16 Conti P, Reale M, Barbacane RC, Castellani ML, Orso C: Differential production of RANTES and MCP-1 in synovial fluid from the inflamed human knee. Immunol Lett 2002;80:105-111.

- 17 Villiger PM, Terkeltaub R, Lotz M: Production of monocyte chemoattractant protein-1 by inflamed synovial tissue and cultured synoviocytes. J Immunol 1992;149:722-727.

18 Melgarejo E, Medina MA, Sanchez-Jimenez F, Urdiales JL: Monocyte chemoattractant protein-1: a key mediator in inflammatory processes. Int J Biochem Cell Biol 2009;41:998-1001.

19 Yuan GH, Masuko-Hongo K, Sakata M, Tsuruha J, Onuma H, Nakamura H, Aoki H, Kato T, Nishioka K: The role of C-C chemokines and their receptors in osteoarthritis. Arthritis Rheum 2001;44:1056-1070.

20 Steppan CM, Bailey ST, Bhat S, Brown EJ, Banerjee RR, Wright CM, Patel HR, Ahima RS, Lazar MA: The hormone resistin links obesity to diabetes. Nature 2001;409:307-312.

21 Reilly MP, Lehrke M, Wolfe ML, Rohatgi A, Lazar MA, Rader DJ: Resistin is an inflammatory marker of atherosclerosis in humans. Circulation 2005;111:932-939.

22 Su CM, Hsu CJ, Tsai CH, Huang CY, Wang SW, Tang CH: Resistin Promotes Angiogenesis in Endothelial Progenitor Cells Through Inhibition of MicroRNA206: Potential Implications for Rheumatoid Arthritis. Stem Cells 2015;33:2243-2255.

23 Wang L, Tang CH, Lu T, Sun Y, Xu G, Huang CC, Yang SF, Su CM: Resistin polymorphisms are associated with rheumatoid arthritis susceptibility in Chinese Han subjects. Medicine (Baltimore) 2018;97:e0177. 


\section{Cellular Physiology Cell Physiol Biochem 2019;52:408-420 \begin{tabular}{cc|c|c|}
\hline DOI: 10.33594/000000029 & (0 2019 The Author(s). Published by \\
\hline
\end{tabular} and Biochemistry Published online: 8 March 2019 Cell Physiol Biochem Press GmbH\&Co. KG \\ Chen et al.: Resistin Enhances MCP-1-Mediated Monocyte Migration}

24 Koskinen A, Vuolteenaho K, Moilanen T, Moilanen E: Resistin as a factor in osteoarthritis: synovial fluid resistin concentrations correlate positively with interleukin 6 and matrix metalloproteinases MMP-1 and MMP-3. Scand J Rheumatol 2014;43:249-253.

- 25 Su CM, Huang CY, Tang CH: Characteristics of resistin in rheumatoid arthritis angiogenesis. Biomark Med 2016;10:651-660.

26 Tang CH, Hsu CJ, Fong YC: The CCL5/CCR5 axis promotes interleukin-6 production in human synovial fibroblasts. Arthritis Rheum 2010;62:3615-3624.

27 Wu MH, Tsai CH, Huang YL, Fong YC, Tang CH: Visfatin Promotes IL-6 and TNF-alpha Production in Human Synovial Fibroblasts by Repressing miR-199a-5p through ERK, p38 and JNK Signaling Pathways. Int J Mol Sci 2018;19:pii:E190.

28 Chen PC, Tang CH, Lin LW, Tsai CH, Chu CY, Lin TH, Huang YL: Thrombospondin-2 promotes prostate cancer bone metastasis by the up-regulation of matrix metalloproteinase- 2 through down-regulating miR-376c expression. J Hematol Oncol 2017;10:33.

29 Chen CY, Su CM, Hsu CJ, Huang CC, Wang SW, Liu SC, Chen WC, Fuh LJ, Tang CH: CCN1 Promotes VEGF Production in Osteoblasts and Induces Endothelial Progenitor Cell Angiogenesis by Inhibiting miR-126 Expression in Rheumatoid Arthritis. J Bone Miner Res 2017;32:34-45.

$30 \mathrm{Hu}$ SL, Chang AC, Huang CC, Tsai CH, Lin CC, Tang CH: Myostatin Promotes Interleukin-1beta Expression in Rheumatoid Arthritis Synovial Fibroblasts through Inhibition of miR-21-5p. Front Immunol 2017;8:1747.

31 Liu SC, Chiu CP, Tsai CH, Hung CY, Li TM, Wu YC, Tang CH: Soya-cerebroside, an extract of Cordyceps militaris, suppresses monocyte migration and prevents cartilage degradation in inflammatory animal models. Sci Rep 2017;7:43205.

32 Liu SC, Hsu CJ, Fong YC, Chuang SM, Tang CH: CTGF induces monocyte chemoattractant protein-1 expression to enhance monocyte migration in human synovial fibroblasts. Biochim Biophys Acta 2013;1833:1114-1124.

33 Tsai CH, Liu SC, Wang YH, Su CM, Huang CC, Hsu CJ, Tang CH: Osteopontin inhibition of miR-129-3p enhances IL-17 expression and monocyte migration in rheumatoid arthritis. Biochim Biophys Acta Gen Subj 2017;1861:15-22.

34 Popolo A, Pinto A, Daglia M, Nabavi SF, Farooqi AA, Rastrelli L: Two likely targets for the anti-cancer effect of indole derivatives from cruciferous vegetables: PI3K/Akt/mTOR signalling pathway and the aryl hydrocarbon receptor. Semin Cancer Biol 2017;46:132-137.

35 Tzeng HE, Tang CH, Wu SH, Chen HT, Fong YC, Lu YC, Chen WC, Huang HD, Lin CY, Wang SW: CCN6-mediated MMP-9 activation enhances metastatic potential of human chondrosarcoma. Cell Death Dis 2018;9:955.

36 Zhang Y, Han JJ, Liang XY, Zhao L, Zhang F, Rasouli J, Wang ZZ, Zhang GX, Li X: miR-23b Suppresses Leukocyte Migration and Pathogenesis of Experimental Autoimmune Encephalomyelitis by Targeting CCL7. Mol Ther 2018;26:582-592.

37 Tsai CH, Tsai HC, Huang HN, Hung CH, Hsu CJ, Fong YC, Hsu HC, Huang YL, Tang CH: Resistin promotes tumor metastasis by down-regulation of miR-519d through the AMPK/p38 signaling pathway in human chondrosarcoma cells. Oncotarget 2015;6:258-270.

38 Tang CH, Chiu YC, Tan TW, Yang RS, Fu WM: Adiponectin enhances IL-6 production in human synovial fibroblast via an AdipoR1 receptor, AMPK, p38, and NF-kappa B pathway. J Immunol 2007;179:5483-5492.

39 Chen HT, Tsou HK, Chen JC, Shih JM, Chen YJ, Tang CH: Adiponectin enhances intercellular adhesion molecule-1 expression and promotes monocyte adhesion in human synovial fibroblasts. PLoS One 2014;9:e92741.

40 Yang WH, Tsai CH, Fong YC, Huang YL, Wang SJ, Chang YS, Tang CH: Leptin induces oncostatin M production in osteoblasts by downregulating miR-93 through the Akt signaling pathway. Int J Mol Sci 2014;15:1577815790.

41 Tong KM, Shieh DC, Chen CP, Tzeng CY, Wang SP, Huang KC, Chiu YC, Fong YC, Tang CH: Leptin induces IL-8 expression via leptin receptor, IRS-1, PI3K, Akt cascade and promotion of NF-kappaB/p300 binding in human synovial fibroblasts. Cell Signal 2008;20:1478-1488.

- 42 Wu C, Qiu S, Liu P, Ge Y, Gao X: Rhizoma Amorphophalli inhibits TNBC cell proliferation, migration, invasion and metastasis through the PI3K/Akt/mTOR pathway. J Ethnopharmacol 2017;211:89-100.

43 Zou W, Ding F, Niu C, Fu Z, Liu S: Brg1 aggravates airway inflammation in asthma via inhibition of the PI3K/ Akt/mTOR pathway. Biochem Biophys Res Commun 2018;503:3212-3218. 


\section{Cellular Physiology Cell Physiol Biochem 2019;52:408-420}

\begin{tabular}{ll|l} 
and Biochemistry & $\begin{array}{l}\text { DOl: 10.33594/000000029 } \\
\text { Published online: } 8 \text { March 2019 }\end{array}$ & $\begin{array}{l}\text { O } 2019 \text { The Author(s). Published by } \\
\text { Cell Physiol Biochem Press GmbH\&Co. KG }\end{array}$ \\
\cline { 2 - 3 }
\end{tabular}

Chen et al.: Resistin Enhances MCP-1-Mediated Monocyte Migration

- 44 Wu MH, Lo JF, Kuo CH, Lin JA, Lin YM, Chen LM, Tsai FJ, Tsai CH, Huang CY, Tang CH: Endothelin-1 promotes MMP-13 production and migration in human chondrosarcoma cells through FAK/PI3K/Akt/mTOR pathways. J Cell Physiol 2012;227:3016-3026.

- 45 Zhang M, Lygrisse K, Wang J: Role of MicroRNA in Osteoarthritis. J Arthritis 2017;6:pii:239.

- 46 Vicente R, Noel D, Pers YM, Apparailly F, Jorgensen C: Deregulation and therapeutic potential of microRNAs in arthritic diseases. Nat Rev Rheumatol 2016;12:211-220. 